\title{
Mg-doped Nickel Oxide as Efficient Hole-Transport Layer for Perovskite Photodetector
}

Lixiang Huang, ${ }^{\text {a }}$ Yukun Wang, ${ }^{\text {a }}$ Xinglin Zhu, ${ }^{\text {a }}$ Xinyu Zhao, ${ }^{\text {a Guoxin }} \mathrm{Li},{ }^{\text {a }}$ Lei Li ${ }^{\mathrm{a}}$ and Wenhong Sun*ab

a Research Center for Optoelectronic Materials and Devices, School of Physical Science and Technology, Guangxi University, Nanning 530004, China.

b Guangxi Key Laboratory of Processing for Non-ferrous Metal and Featured Materials, Guangxi University, Nanning 530004, China.

*corresponding e-mail address: ykwang0929@163.com (Yukun Wang); 20180001@gxu.edu.cn (Wenhong Sun) 


\section{Supporting Information}
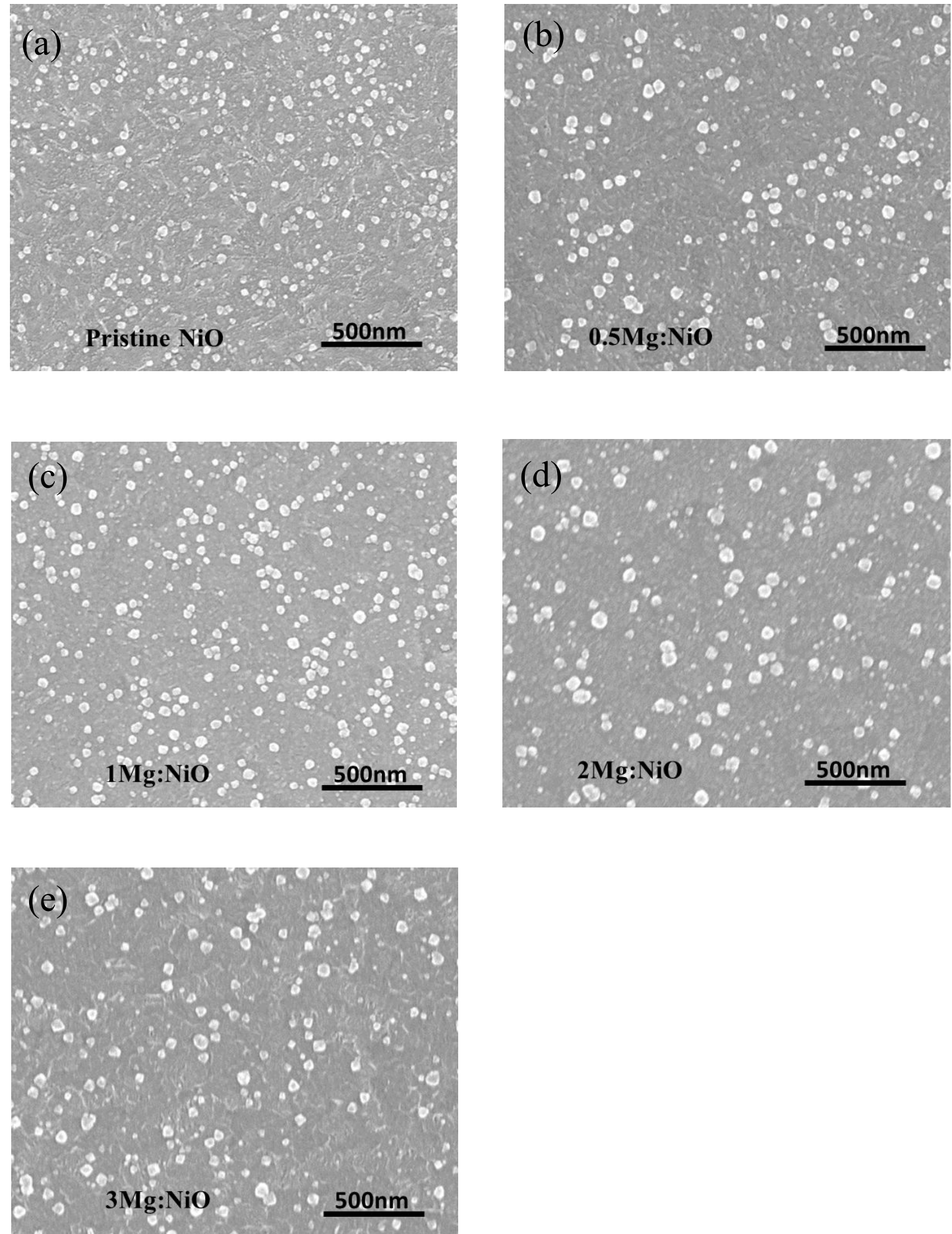

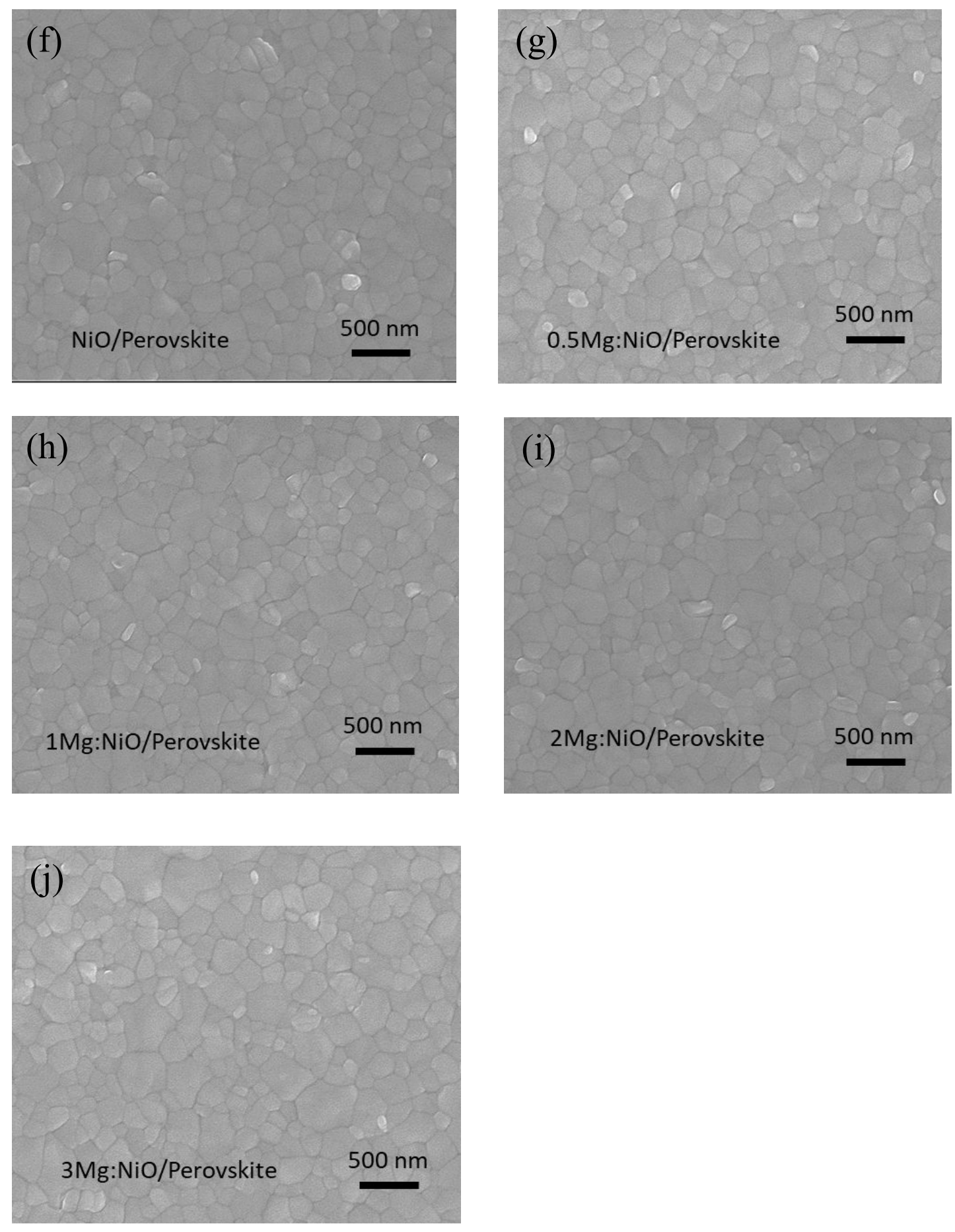

Figure S1. SEM images of (a) pure $\mathrm{NiO}$, (b) $0.5 \mathrm{Mg}: \mathrm{NiO}$, (c) $1 \mathrm{Mg}: \mathrm{NiO}$, (d) $2 \mathrm{Mg}: \mathrm{NiO}$, (e) $3 \mathrm{Mg}: \mathrm{NiO}$ and perovskites formed on (f) pure $\mathrm{NiO}$, (g) $0.5 \mathrm{Mg}: \mathrm{NiO}$ (h) $1 \mathrm{Mg}: \mathrm{NiO}$, (i) 2Mg:NiO, (j) 3Mg:NiO. 
(a)

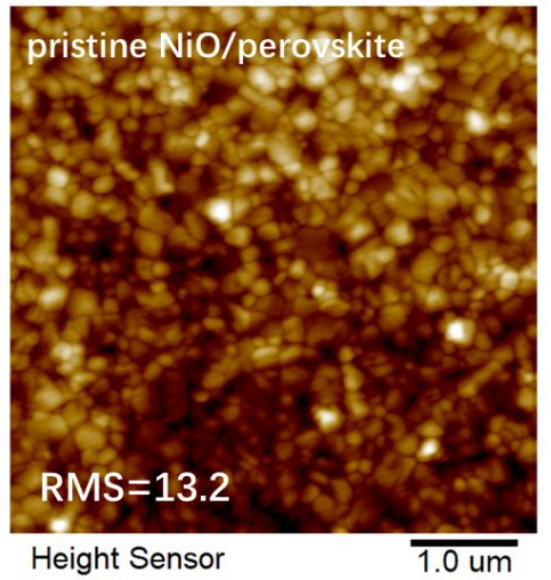

(c)

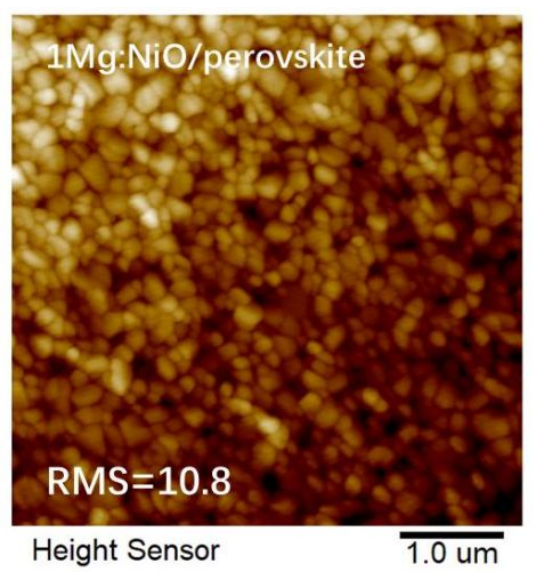

(e)

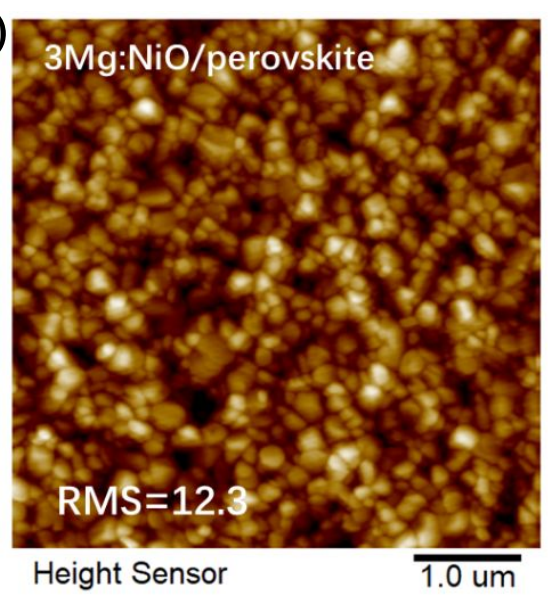

(b)

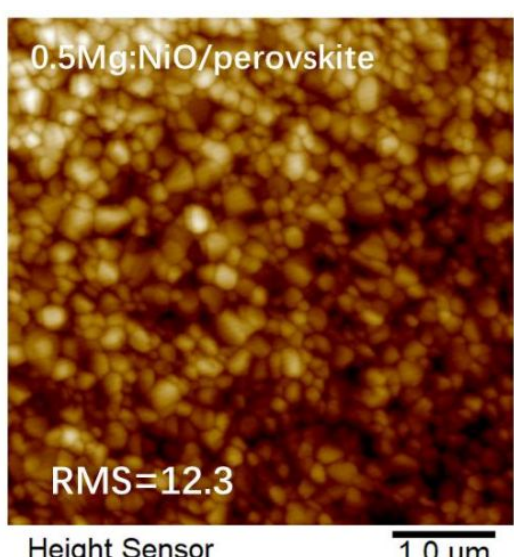

$366.4 \mathrm{~nm}$

$608.7 \mathrm{~nm}$

$501.5 \mathrm{~nm}$

Height Sensor

$\overline{1.0 \mathrm{um}}$

(d)

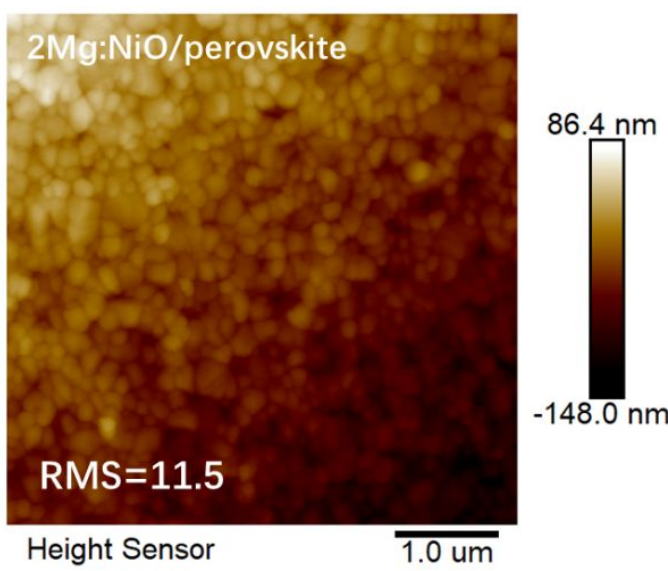

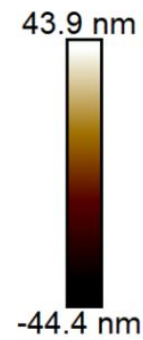

Figure S2. AFM images of perovskite film formed on (a) pure $\mathrm{NiO}$, (b) $0.5 \mathrm{Mg}: \mathrm{NiO}$, (c) $1 \mathrm{Mg}: \mathrm{NiO}$, (d) $2 \mathrm{Mg}: \mathrm{NiO}$, (e) $3 \mathrm{Mg}: \mathrm{NiO}$ 


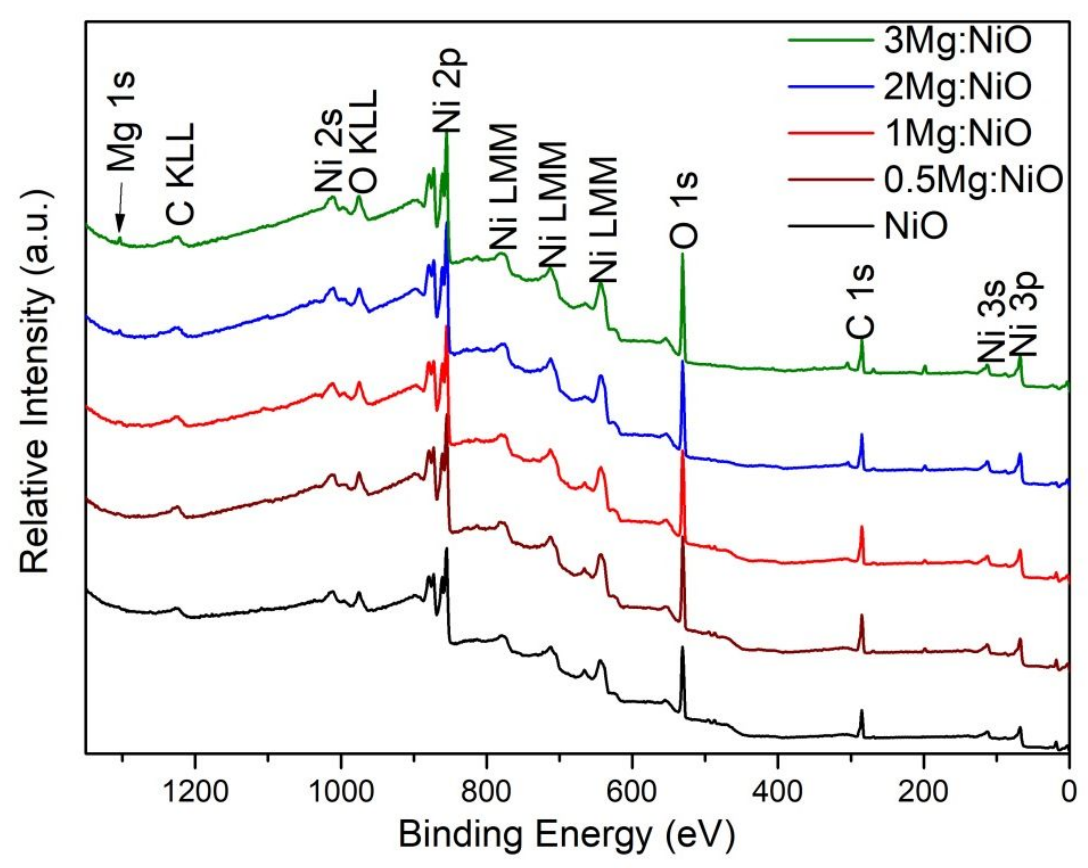

Figure S3. Wide survey XPS spectra of different HTLs.

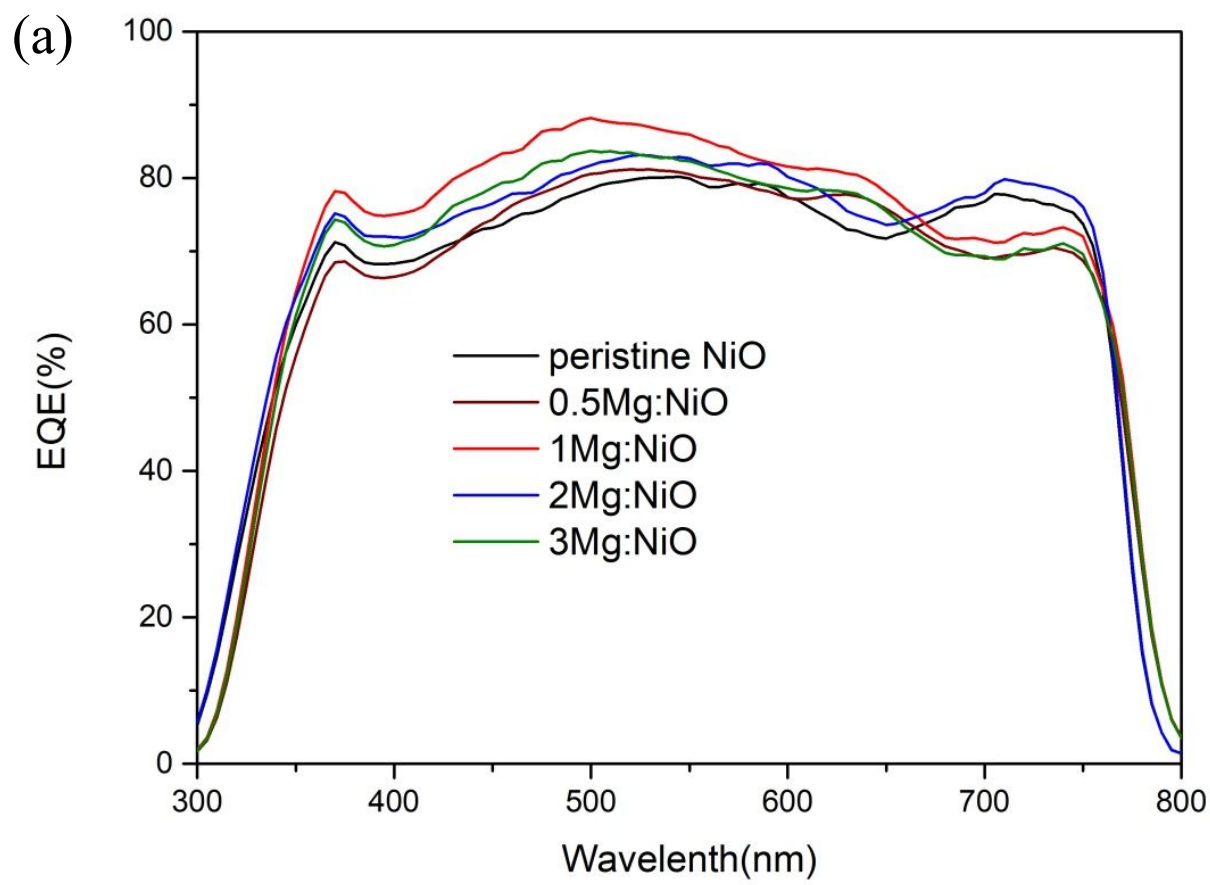




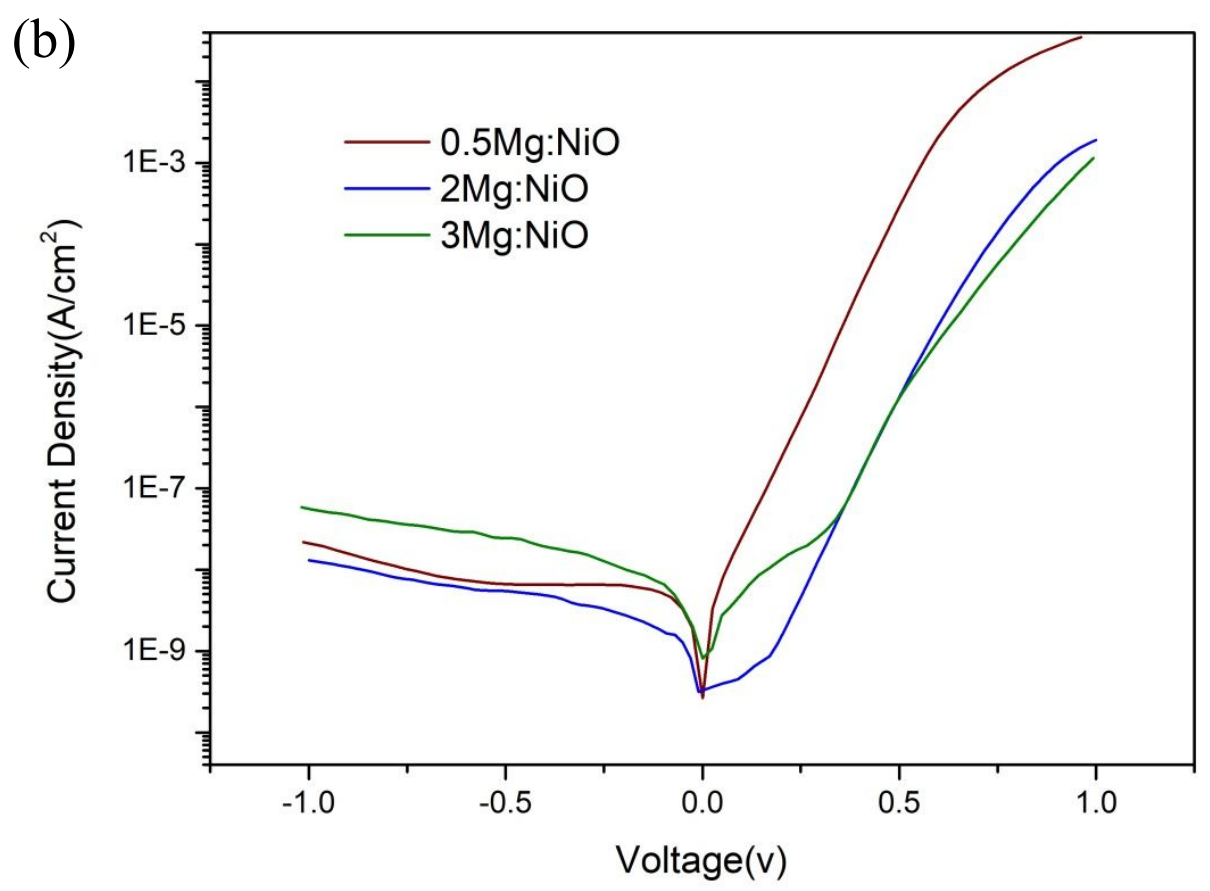

(c)

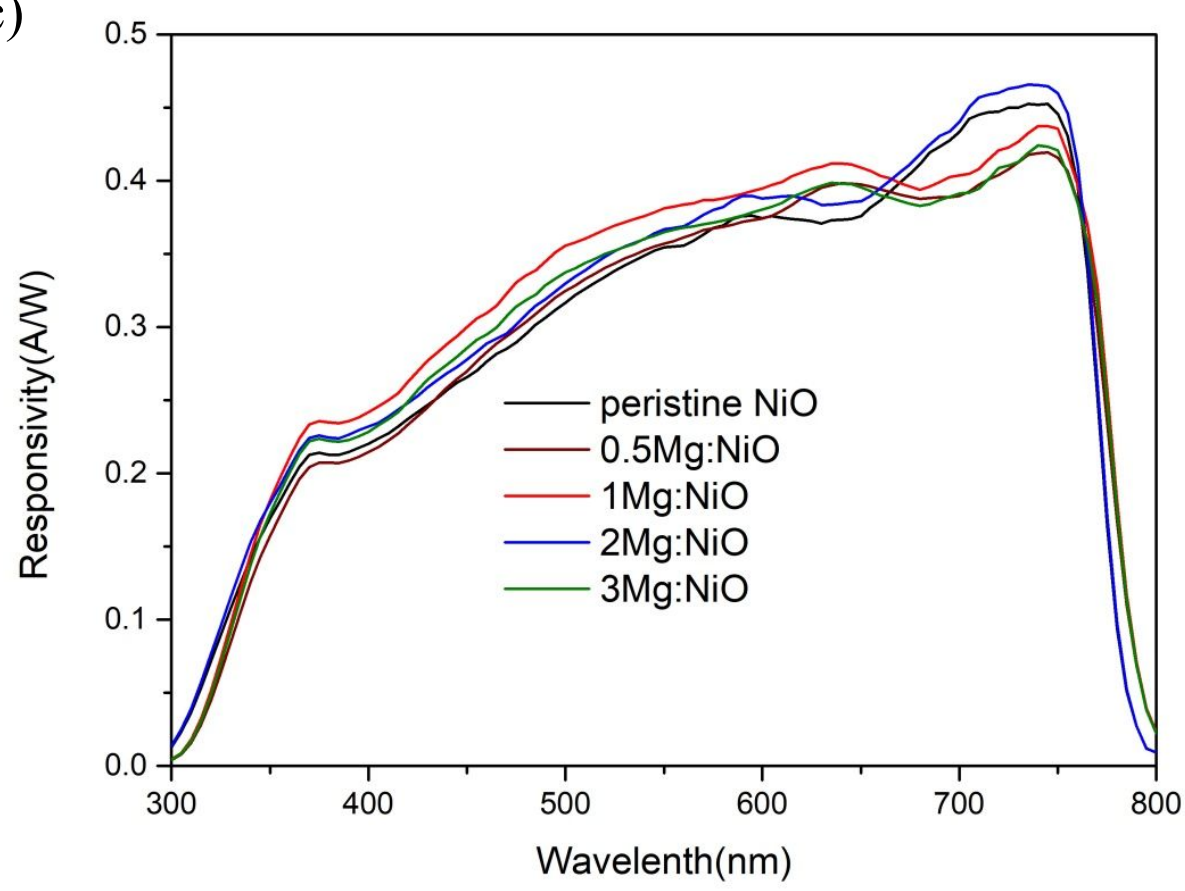

Figure S4. (a) EQE of the PDs using different HTLs. (b) Dark current density-voltage characteristics of the PDs based on different HTLs. (c) Responsivity of the PDs based on $\mathrm{NiO}$ anode interlayers with and without added $\mathrm{Mg}$ at different wavelengths. 